\title{
Miniscrew appliances and their use in orthodontics
}

\author{
Rocío Molina-Solana, R. M. Yáñez-Vico, Alejandro Iglesias-Linares, Daniel Torres-Lagares, \\ Enrique Solano-Reina \\ Department of Stomatology, University of Seville, Seville, Spain \\ Email: rosayanezvico@gmail.com
}

Received 31 January 2013; revised 28 February 2013; accepted 14 March 2013

\begin{abstract}
Anchorage is an important factor in orthodontics and must be properly managed for a successful treatment outcome. Orthodontists have historically used many devices to prevent unwanted tooth movement. The use of miniscrews as a source of skeletal anchorage has become routine in present-day orthodontic practice. Skeletal anchorage opens up alternative treatments, to borderline surgery patients for example, who can now be treated by taking advantage of the biomechanical properties of miniscrews. Because they are bone-borne and provide excellent control over tooth movement in the three spatial planes of movement, mini-implants can be used to serve many purposes in addition to anchorage. Therefore, a variety of successful mini-implant appliances are explained in relation to the three planes of movement: sagittal, vertical, and transverse. The aim of this review is to illustrate the clinical versatility of miniscrews in the three spatial planes of malocclusion in orthodontic and orthopedic management and to define clinical guidelines for the selection, application and biomechanics of mini-implants.
\end{abstract}

Keywords: Miniscrew; Mini-implant; Orthodontics; Anchorage

\section{INTRODUCTION}

Anchorage control in orthodontic treatment is an important factor in determining treatment outcome. Orthodontic therapy has traditionally used the teeth and extraoral and/or intermaxillary appliances to guard against loss of anchorage. However, many of these devices call for patient cooperation, and loss of anchorage is often observed nevertheless.

Miniscrews were introduced as absolute anchorage devices in orthodontic treatment [1] because the procedures for placing and removing are less invasive than for other implants and they do not require patient cooperation. The miniscrew is anchored in the bone and then removed after completion of orthodontic tooth movement. Excellent treatment results have been reported using miniscrews for orthodontic anchorage in different types of malocclusion [2], since they provide stable anchorage for different tooth movements, including intrusion, extrusion, distalization, protraction, midline coordination and changes in the occlusal plane. It is necessary to provide an exhaustive orthodontic diagnosis and select miniscrews of the correct length and diameter as well as the distribution of orthodontic forces. A judicious combination of these factors can provide excellent results in the treatment of malocclusions, by increasing anchorage, and moving and controlling the teeth and dentoalveolar process in all three planes of space.

However, various problems have been reported in relation to their use, including a high failure rate, due to factors such as gingival inflammation, the diameter of the miniscrew, the site of implantation, or the proximity of the device to the root $[3,4]$. Successful placement, on the other hand, depends on the optimum design, shape and size of the miniscrew, the angle of insertion and the size of the anatomical area where the insertion is to be made. So, for the technique to be successful, a combination of factors must be taken into account.

In this article, we develop treatment using miniscrews with the aim of avoiding unwanted outcomes. Therefore, a variety of successful mini-implant appliances are explained in relation to the three planes of movement: sagittal, vertical, and transverse.

\section{CONSIDERATIONS FOR INSERTION}

To achieve a successful miniscrew placement, there are guidelines for insertion which will help prevent screw loss or potential root damage and ensure a better treatment outcome. The aim of the technique we describe here is to make screw insertion safer and is based on the rule of complete asepsis, a careful anesthesia technique, reference landmark guidelines, and the safe insertion of the screw itself [5].

The patient should use mouthwash or disinfectant, such as chlorhexidine. An antibacterial, such as chlor- 
hexidine digluconate, should also be applied to the area where the miniscrew is to be placed to maintain asepsis. Placement should take place under local anesthesia, with just a small injection inside the mouth being sufficient to achieve the anesthetic effect. If the patient mentions sensitivity while the miniscrew is being inserted, this may be because the miniscrew has made contact with a tooth root [6]. However, perforation can be avoided by considering redirecting the screw. Careful planning and radiographic evaluation of the placement site can minimize this risk. Before inserting the miniscrew, the inexperienced orthodontist is advised to use a periapical $\mathrm{x}$-ray in conjunction with a bent guidewire in the area selected for the insertion. Fabrication of a bar to serve as a guide is another option (Figure 1). These landmarks may remain during the surgery. The experienced orthodontist, however, will be able to place a miniscrew without such landmark guides [1]. Miniscrew insertion should take into account the relationship between the interradicular bone and the length of the screw to be embedded in the cortical bone. A miniscrew placed near the cementoenamel junction has less interradicular space available than one inserted near the tooth apex instead. For optimal retention and effectiveness, the screw should be inserted with a $5 \mathrm{~mm}$ inclination towards the cementoenamel junction and at an angle of more than $45^{\circ}$ towards the tooth apex [3,7].

In the mandible, lingual screw insertion is associated with higher loss rates, and the mentalis region is more suitable when miniscrews are used $[5,6]$. In the maxilla, the anterior palate appears to be one of the best locations for orthodontic miniscrews because the cortical bone is thicker there than at other insertion sites [6]. Miniscrews vary from $1-2.3 \mathrm{~mm}$ in diameter and $4-21 \mathrm{~mm}$ in length. To avoid damaging the root or the periodontium, screw size should be selected to match the chosen insertion site, bearing in mind that a screw with a smaller diameter is more susceptible to fracture when being placed. Burnstone et al. [5] reported that success rates for mini-
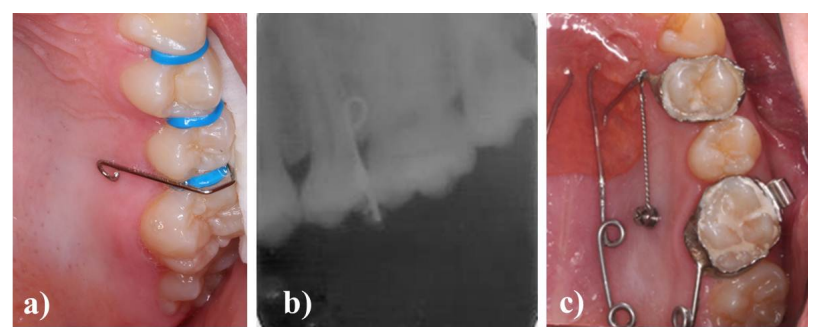

Figure 1. $1.6 \times 10 \mathrm{~mm}$ miniscrew ACR $(\mathrm{H})$ model (Jeil Medical Corp, Korea) inserted into the palatal region. (a) Bent wire guideline located in the insertion area; (b) Periapical radiograph in association with a bent wire located in the chosen insertion area used to ensure proper insertion of the miniscrew; (c) Upper molar distalization obtained using a miniscrew combined with an intraoral distalizer (hexahelix). screws of $1.2 \mathrm{~mm}$ diameter and at least $8 \mathrm{~mm}$ long were above $70 \%$, and concluded that not placing a screw perpendicular to the bone surface reduced the risk of damaging the root and increased the contact of the screw with the cortical bone.

\section{SAGITTAL PLANE}

The orthodontic effects that can be achieved in the sagittal plane using miniscrews are referred to in the literature as upper and lower molar distalization, molar protraction, midline coordination and canine distalization. These movements are indicated to correct class II or III malocclusions: retraction of anterior teeth following extraction of first molars or deviation of the maxillary dental midline towards the left or right of the facial midline.

\subsection{Upper Molar Distalization}

The validity of a clinical use of interradicular miniscrew anchorage for the distal movement of maxillary molars has been referred to recently in the literature. Yamada et al. [8] concluded that miniscrews placed in the maxillary interradicular space provided successful distal movement of molars without patient cooperation or undesirable side effects. Palatal implants have also been used for molar distalization. Gelgor et al. [9] provided anchorage and indirect distalization with a miniscrew in the palate that connected a transpalatal bar to an upper first premolar on either side. Other authors [10,11] have also used similar systems, with miniscrews supported by auxiliary elements, such as an intraoral distalizer or transpalatal bar, in order to gain direct or indirect anchorage. So, both anchorage and distalization can be obtained by using miniscrews with an auxiliary element [9-12], with successful distalization rates and no loss of anchorage (Figure 1).

\subsection{Lower Molar Distalization}

Lower molar distalization has also been described in the literature. In 2005, Chung et al. [13] used miniscrews for indirect anchorage in the lower dentition. Two miniscrews were inserted between the upper premolars and molars, and Class III elastics run through them to achieve successful distalization. A further possibility is a mini-implant inserted in the ramus of the mandible (Figure 2) providing direct anchorage for lower dentition distalization with a high rate of success (Figure 3).

\subsection{Midline Coordination}

Another of the applications of miniscrew appliances seems to be to provide anchorage for midline coordination. In their 2011 study, Yanagita et al. [2] placed a miniscrew in the right buccal area following the closure 

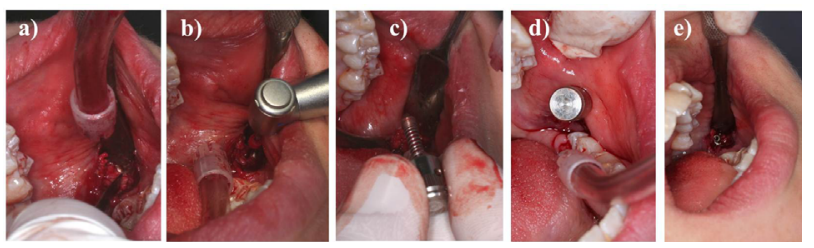

Figure 2. $1.6 \times 8$ mm miniscrew, ACR $(\mathrm{H})$ model (Jeil Medical Corp, Korea) was placed in the mandibular ramus for lower molar distalization. (a) Soft tissue incision; (b) Previous drilling; (c), (d) Bar guide to ensure proper insertion of the miniscrew; (e) Final right-hand thread of the miniscrew.

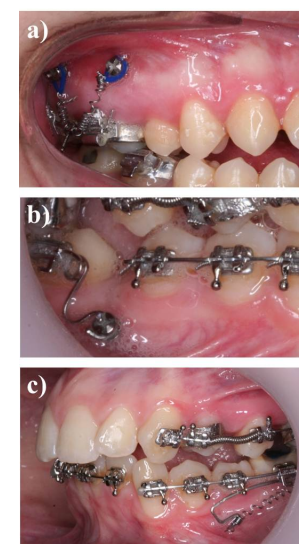

Figure 3. Successful dental movements in the three planes of movement achieved with miniscrew anchorage. (a) Vertical: posterior intrusion achieved by placing two miniscrews both mesial and distal to the upper molar with a closed coil-spring and transpalatal bar. $1.6 \times 8$ mm miniscrews, ACR (H) model (Jeil Medical Corp, Korea); (b) Rotation: lower molar uprighting and molar rotation: $1.6 \times 8 \mathrm{~mm}$ miniscrew ACR $(\mathrm{H})$ (Jeil Medical Corp, Korea); (c) Sagittal: miniscrew placed in the mandibular ramus for lower molar distalization. $1.6 \times$ $8 \mathrm{~mm}$ miniscrew, ACR (H) model (Jeil Medical Corp, Korea).

of the extraction space in order to correct the maxillary midline. In that case, mandibular molar uprighting was also required. The result was alignment of the dental midline with the facial midline and molar uprighting.

\subsection{Canine Retraction}

In 2011, Aboul-Ela et al. [14] published a study evaluating treatment using a miniscrew implant to support maxillary canine retraction following extraction of the maxillary first premolars. This study reported no molar anchorage loss during canine retraction, indicating that miniscrew anchorage could be successfully used for this purpose.

Thiruvenkatachari et al. [15] compared and measured the amount of anchorage loss during canine retraction using both miniscrews and conventional molar anchorage. Their results showed no anchorage loss on the miniscrew side and they concluded that miniscrews could be used for canine retraction with a high success rate. Many other authors [16-18] have also published studies on the retraction of anterior teeth using miniscrews. The extraction space was fully utilized during canine retraction with no anchorage loss.

\subsection{Molar Protraction}

Freudenthaler et al. [19] studied the effectiveness of miniscrews in protracting the lower molars into the extraction socket. Miniscrews $2 \mathrm{~mm}$ in diameter were inserted between the second premolars at the level of the apical thirds. Similarly, Kyung et al. [20] reported the case of a patient whose lower first molars had been extracted. The lower second molars were protracted with the aid of miniscrews placed between the premolars. Giancotti et al. [21] also illustrated their clinical experience of an adult patient who was treated by extracting the mandibular first molars and placing miniscrews distal to the second premolars. The result was space closure with no anchorage loss and successful protraction of the second and third molars into the extraction sites.

\section{VERTICAL PLANE}

Intrusion of posterior and anterior teeth is always difficult to achieve without the side effect of extrusion of the anchorage teeth; the use of miniscrews for skeletal anchorage provides a solution without the adverse effects.

\subsection{Canine and Incisor Extrusion}

In 2010, Kocsis et al. [22] published a study evaluating the possible effect of miniscrews in managing impacted upper canines. An intraosseous screw was placed into the premolar-molar interradicular space. A high rate of success was achieved and they concluded that miniscrew anchorage should be borne in mind when planning the extrusion of impacted canines, in line with previous studies [23]. Where there are prosthodontic requirements, miniscrews can also be used to handle canine extrusion. Roth et al. [24] reported a case where incisor and canine extrusion was needed for the future construction of a bridge. The patient's right incisor was missing and the gingiva at the central incisor and canine had receded by 3 $4 \mathrm{~mm}$. The miniscrew was placed into the alveolus of the missing upper lateral incisor, and extrusion of central incisor and canine was successfully achieved.

\subsection{Molar Intrusion}

Miniscrews are very useful for the non-surgical manage- 
ment of anterior open bite cases with molar intrusion (Figure 3). A recent 2010 case report [25] described the successful treatment of a severe anterior open bite using miniscrew anchorage. The miniscrews were implanted bilaterally between the second premolar and the first molar, and an intrusion force applied. The results showed that the maxillary molars were intruded and a good occlusion achieved. Another case report [26] from 2009 reported the case of an open bite treated by intruding the molars with four miniscrews placed bilaterally in the interradicular space between both upper and lower posterior teeth. Upper molar intrusion is also illustrated in Figure 3 , where anchorage was achieved by inserting two miniscrews mesial and distal to the upper first molar using a closed coil-spring and transpalatal bar.

Overeruption of maxillary molars resulting from the loss of the opposite teeth is frequently seen in clinical practice today. Overeruption interferes with occlusion and creates functional disturbances. In order to restore a proper occlusion, intrusion of overerupted molars is essential. Many studies have been published which have concluded that the miniscrew appliance can be successfully used for molar intrusion. In one recent report [27], the authors described maxillary molar intrusion in order to gain enough prosthetic space for a future implant. Other studies have been published in which overerupted lower and upper molars were intruded with miniscrews [28]. Upper molars can also be intruded with miniscrews on the buccal and palatal sides before commencing the prosthetic restoration of missing teeth $[29,30]$.

\section{TRANSVERSE PLANE}

For patients with severe transverse discrepancies, miniscrews can facilitate rapid palatal expansion, using Hyrax-type rapid palatal expanders combined with orthodontic miniscrews to achieve a successful transverse correction [31,32]. In mild Class III cases, miniscrews may also be a suitable alternative to extraoral appliances, and possibly surgery. The case of a 12-year-old boy treated for maxillary deficiency with miniscrew implants has been illustrated in the literature [33]. The miniscrews were inserted between the canines and first premolars under local anesthesia, as previously seen, before being treated with Class III elastics running from two mandibular miniscrew implants to a removable upper appliance. Both orthopedic and orthodontic effects were achieved.

\section{ROTATIONAL MOVEMENTS}

\subsection{Changes in the Occlusal Plane}

Conventionally, a combination of mandibular and maxillary osteotomy has been used to correct both mandibular deviation and canted maxillary occlusal plane. One case, reported by Hashimoto et al. [34], suggested a combination treatment using miniscrews and mandibular osteotomy, and the authors described the functional and morphologic changes achieved using miniscrew anchorage instead of maxillary osteotomy. The canted maxillary occlusal plane was corrected using miniscrew anchorage for intrusion of the maxillary right molars, and the mandibular deviation by mandibular osteotomy. The authors concluded that the combination of miniscrew anchorage and mandibular osteotomy was effective for treating patients with mandibular deviation and canted maxillary occlusal plane. In 2006, DeVincenzo [35] presented another case report about correction of the occlusal plane. The author developed a new appliance, called the vertical adjustable corrector, to treat extreme dolichocephalic malocclusions. Intrusion of posterior or anterior teeth may alter the occlusal plane and their conclusion was that this method represented an alternative to surgery.

\subsection{Molar Uprighting}

Different biomechanics have been reported for molar uprighting. Park et al. [36,37] reported that miniscrews could be used to upright mesially inclined mandibular and maxillary second molars. Elastic thread was needed to achieve the effect, and upright the mesially angulated second molar. In 2011 Yanagita et al. [2] used miniscrew anchorage to upright lower molars and correct severe crowding and a midline deviation. The results were retraction of the mandibular teeth into the extraction socket with absolute anchorage and correction of a Class III malocclusion and dental midline deviation. Giancotti et al. [38] uprighted an impacted mandibular second molar with a closed-coil spring, attached to a miniscrew implanted in the retromolar region. In 2005, Yun et al. [39] described the use of miniscrews to provide indirect anchorage when uprighting two cases of mesially tipped mandibular second molars.

\section{CONCLUSION}

The introduction of miniscrews into orthodontics has had a revolutionary impact on this specialty. Retraction or protraction of the whole dentition in the anteroposterior direction, absolute intrusion of posterior teeth in the vertical direction, and palatal expansion can now be achieved using miniscrews. The aim of this review was to define clinical guidelines for mini-implant selection, application and biomechanics, as well as illustrate their different applications in the three spatial planes.

\section{REFERENCES}

[1] Leung, M.T., Lee, T.C., Rabie, A.B. and Wong, R.W. (2008) Use of miniscrews and miniplates in orthodontics. 
Journal of Oral and Maxillofacial Surgery, 66, 14611466. doi:10.1016/j.joms.2007.12.029

[2] Yanagita, T., Kuroda, S., Takano-Yamamoto, T. and Yamashiro, T. (2011) Class III malocclusion with complex problems of lateral open bite and severe crowding successfully treated with miniscrew anchorage and lingual orthodontic brackets. American Journal of Orthodontics and Dentofacial Orthopedics, 139, 679-689. doi:10.1016/j.ajodo.2009.07.023

[3] Moon, C.H., Lee, D.G., Lee, H.S., Im, J.S. and Baek, S.H. (2008) Factors associated with the success rate of orthodontic miniscrews placed in the upper and lower posterior buccal region. Angle Orthodontist, 78, 101-106. doi:10.2319/121706-515.1

[4] Topouzelis, N. and Tsaousoglou, P. (2012) Clinical factors correlated with the success rate of miniscrews in orthodontic treatment. International Journal of Oral Science, 4, 38-44.

[5] Crismani, A.G., Bertl, M.H., Celar, A.G., Bantleon, H.P. and Burstone, C.J. (2010) Miniscrews in orthodontic treatment: Review and analysis of published clinical trials. American Journal of Orthodontics and Dentofacial Orthopedics, 137, 108-113. doi:10.1016/j.ajodo.2008.01.027

[6] Ludwig, B., Glasl, B., Bowman, S.J., Wilmes, B., Kinzinger, G.S. and Lisson, J.A. (2011) Anatomical guidelines for miniscrew insertion: Palatal sites. Journal of Clinical Orthodontics, 45, 433-441.

[7] Suzuki, A., Masuda, T., Takahashi, I., Deguchi, T., Suzuki, O. and Takano-Yamamoto, T. (2011) Changes in stress distribution of orthodontic miniscrews and surrounding bone evaluated by 3-dimensional finite element analysis. American Journal of Orthodontics and Dentofacial Orthopedics, 140, 273-280. doi:10.1016/j.ajodo.2011.06.025

[8] Yamada, K., Kuroda, S., Deguchi, T., Takano-Yamamoto, T. and Yamashiro, T. (2009) Distal movement of maxillary molars using miniscrew anchorage in the buccal interradicular region. Angle Orthodontist, 79, 78-84. doi:10.2319/020408-68.1

[9] Gelgor, I.E., Buyukyilmaz, T., Karaman, A.I., Dolanmaz, D. and Kalayci, A. (2004) Intraosseous screw-supported upper molar distalization. Angle Orthodontist, 74, 838850.

[10] Kyung, S.H., Hong, S.G. and Park, Y.C. (2003) Distalization of maxillary molars with a midpalatal miniscrew. Journal of Clinical Orthodontics, 37, 22-26.

[11] Kinzinger, G.S., Gülden, N., Yildizhan, F. and Diedrich, P.R. (2009) Efficiency of a skeletonized distal jet appliance supported by miniscrew anchorage for noncompliance maxillary molar distalization. American Journal of Orthodontics and Dentofacial Orthopedics, 136, 578-586. doi:10.1016/j.ajodo.2007.10.049

[12] Lim, J.K., Jeon, H.J. and Kim, J.H. (2011) Molar distalization with a miniscrew-anchored sliding jig. Journal of Clinical Orthodontics, 45, 368-377.

[13] Chung, K., Kim, S.H. and Kook, Y. (2005) C-orthodontic microimplant for distalization of mandibular dentition in Class III correction. Angle Orthodontist, 75, 119-128.

[14] Aboul-Ela, S.M., El-Beialy, A.R., El-Sayed, K.M., Selim,
E.M., El-Mangoury, N.H. and Mostafa, Y.A. (2011) Miniscrew implant-supported maxillary canine retraction with and without corticotomy-facilitated orthodontics. American Journal of Orthodontics and Dentofacial Orthopedics, 139, 252-259. doi:10.1016/j.ajodo.2009.04.028

[15] Thiruvenkatachari, B., Pavithranand, A., Rajasigamani, K. and Kyung, H.M. (2006) Comparison and measurement of the amount of anchorage loss of the molars with and without the use of implant anchorage during canine retraction. American Journal of Orthodontics and Dentofacial Orthopedics, 129, 551-554. doi:10.1016/j.ajodo.2005.12.014

[16] Park, H.S., Kwon, O.W. and Sung, J.H. (2005) Microscrew implant anchorage sliding mechanics. World Journal of Orthodontics, 6, 265-274.

[17] Lee, J.S., Park, H.S. and Kyung, H.M. (2001) Microimplant anchorage for lingual treatment of a skeletal Class II malocclusion. Journal of Clinical Orthodontics, 35, 643-647.

[18] Bae, S.M., Park, H.S., Kyung, H.M., et al. (2002) Clinical application of micro-implant anchorage. Journal of Clinical Orthodontics, 36, 298-302.

[19] Freudenthaler, J.W., Haas, R. and Bantleon, H.P. (2001) Bicortical titanium screws for critical orthodontic anchorage in the mandible: A preliminary report on clinical applications. Clinical Oral Implants Research, 12, 358363. doi:10.1034/j.1600-0501.2001.012004358.X

[20] Kyung, S.H., Choi, J.H. and Park, Y.C. (2003) Miniscrew anchorage used toprotract lower second molars into first molar extraction sites. Journal of Clinical Orthodontics, 37, 575-579.

[21] Giancotti, A., Greco, M., Mampieri, G. and Arcuri, C. (2004) The use of titanium miniscrews for molar protraction in extraction treatment. Progress in Orthodontics, 5, 236-247.

[22] Kocsis, A., Seres, L., Kocsis-Savanya, G. and Kovács, A. (2010) Skeletal anchorage: Use of miniscrews for impacted maxillary canine management. Fogorvosi Szemle, 103, 3-9.

[23] Park, H.S., Kwon, O.W. and Sung, J.H. (2004) Microimplant anchorage for forced eruption of impacted canines. Journal of Clinical Orthodontics, 38, 297-302.

[24] Roth, A., Yildirim, M. and Diedrich, P. (2004) Forced eruption with microscrew anchorage for preprosthetic leveling of the gingival margin. Case report. Journal of Orofacial Orthopedics, 65, 513-519. doi:10.1007/s00056-004-0430-z

[25] Rachala, M.R. and Harikrishnan, P. (2010) Management of severe anterior open-bite in an adult patient using miniscrews as skeletal anchorage. International Journal of Orthodontics Milwaukee, 21, 9-14.

[26] Kaku, M., Kawai, A., Koseki, H., et al. (2009) Correction of severe open bite using miniscrew anchorage. Australian Dental Journal, 54, 374-380. doi:10.1111/j.1834-7819.2009.01166.x

[27] Bratu, C.D., Pop, R.V., Pop, S.I. and Bratu, E.A. (2011) Adjusting dento-alveolar morphology with orthodontic mini-implants (miniscrews). A clinical case report. Romanian Journal of Morphology and Embryology, 52, 


\section{3-1137.}

[28] Bae, S.M. and Kyung, H.M. (2006) Mandibular molar intrusion with miniscrew anchorage. Journal of Clinical Orthodontics, 40, 107-108.

[29] Lin, J.C.Y., Liou, E.J.W. and Yeh, C.-L. (2006) Intrusion of overerupted maxillary molars with miniscrew anchorage. Journal of Clinical Orthodontics, 40, 378-383.

[30] Chang, Y.J., Lee, H.S. and Chun, Y.S. (2004) Microscrew anchorage for molar intrusion. Journal of Clinical Orthodontics, 38, 325-330.

[31] Lee, K.J., Park, Y.C., Park, J.Y. and Hwang, W.S. (2010) Miniscrew-assisted nonsurgical palatal expansion before orthognathic surgery for a patient with severe mandibular prognathism. American Journal of Orthodontics and Dentofacial Orthopedics, 137, 830-839. doi:10.1016/j.ajodo.2007.10.065

[32] Harzer, W., Schneider, M. and Gedrange, T. (2004) Rapid maxillary expansion with palatal anchorage of the hyrax expansion screw-pilot study with case presentation. Journal of Orofacial Orthopedics, 65, 419-424. doi:10.1007/s00056-004-0346-7

[33] Jamilian, A. and Showkatbakhsh, R. (2010) Treatment of maxillary deficiency by miniscrew implants: A case report. Journal of Orthodontics, 37, 56-61. doi:10.1179/14653121042876
[34] Hashimoto, T., Fukunaga, T., Kuroda, S., Sakai, Y., Yamashiro, T. and Takano-Yamamoto, T. (2009) Mandibular deviation and canted maxillary occlusal plane treated with miniscrews and intraoral vertical ramus osteotomy: functional and morphologic changes. American Journal of Orthodontics and Dentofacial Orthopedics, 136, 868877. doi:10.1016/j.ajodo.2007.06.022

[35] DeVincenzo, J.P. (2006) A new non-surgical approach for treatment of extreme dolichocephalic malocclusions. Part 1. Appliance design and mechanotherapy. Journal of Clinical Orthodontics, 40, 161-170.

[36] Park, H.S., Kwon, O.W. and Sung, J.H. (2004) Uprighting second molars with micro-implant anchorage. Journal of Clinical Orthodontics, 38, 100-103.

[37] Park, H.S., Kyung, H.M. and Sung, J.H. (2002) A simple method of molar uprighting with micro-implant anchorage. Journal of Clinical Orthodontics, 36, 592-596.

[38] Giancotti, A., Arcuri, C. and Barlattani, A. (2004) Treatment of ectopic mandibular second molar with titanium miniscrews. American Journal of Orthodontics and Dentofacial Orthopedics, 126, 113-117. doi:10.1016/j.ajodo.2003.08.025

[39] Yun, S.W., Lim, W.H. and Chun, Y.S. (2005) Molar control using indirect miniscrew anchorage. Journal of Clinical Orthodontics, 39, 661-664. 